\title{
COMPUTING WITH NILPOTENT ORBITS IN SIMPLE LIE ALGEBRAS OF EXCEPTIONAL TYPE
}

\author{
WILLEM A. DE GRAAF
}

Abstract

\begin{abstract}
Let $G$ be a simple algebraic group over an algebraically closed field with Lie algebra $\mathfrak{g}$. Then the orbits of nilpotent elements of $\mathfrak{g}$ under the adjoint action of $G$ have been classified. We describe a simple algorithm for finding a representative of a nilpotent orbit. We use this to compute lists of representatives of these orbits for the Lie algebras of exceptional type. Then we give two applications. The first one concerns settling a conjecture by Elashvili on the index of centralizers of nilpotent orbits, for the case where the Lie algebra is of exceptional type. The second deals with minimal dimensions of centralizers in centralizers.
\end{abstract}

\section{Introduction}

Let $G$ be a simple algebraic group over an algebraically closed field of characteristic 0 . Let $\mathfrak{g}$ denote its Lie algebra. Then $G$ acts on $\mathfrak{g}$ via the adjoint representation. It is a natural question what the $G$-orbits in $\mathfrak{g}$ are. Recall that an element $e \in \mathfrak{g}$ is said to be nilpotent if the map ade $: \mathfrak{g} \rightarrow \mathfrak{g}$ is nilpotent. Now the $G$-orbits of nilpotent elements in $\mathfrak{g}$ are called nilpotent orbits. These have drawn a lot of attention in the past decades. On some occasions it turns out that using conceptual arguments to prove their properties is a lot harder for the exceptional types than it is for the classical types. However, for the former an approach based on a case by case analysis is possible. It is the objective of this paper to describe how this can be carried out using computer calculations.

The nilpotent orbits in $\mathfrak{g}$ are classified in terms of so-called weighted Dynkin diagrams. The first problem that we consider is to find a nilpotent element in $\mathfrak{g}$ given the corresponding weighted Dynkin diagram. We describe a straightforward algorithm for this (Section 3). The algorithm is used to compute lists of explicit representatives of the nilpotent orbits in the Lie algebras of exceptional type. They are listed in Appendix A.

We use these lists to prove Elashvili's conjecture for the exceptional types by computer calculations. This conjecture concerns the index of centralizers of nilpotent elements. The concept of index is defined as follows. Let $K$ be a finite-dimensional Lie algebra, and let $K^{*}$ denote the dual space. For $f \in K^{*}$ set $K^{f}=\{x \in K \mid$ $f([x, y])=0$ for all $y \in K\}$. Then the index of $K$ is defined as the number

$$
\operatorname{ind}(K)=\inf _{f \in K^{*}} \operatorname{dim} K^{f} \text {. }
$$


For semisimple Lie algebras in characteristic zero it is known that the index is equal to the rank ([4], Proposition 1.11.12).

By $C_{\mathfrak{g}}(x)$ we denote the centralizer of of $x \in \mathfrak{g}$.

CONJeCture 1 (Elashvili). Let $\mathfrak{g}$ be a semisimple Lie algebra over an algebraically closed field of characteristic 0 . Let $x \in \mathfrak{g}$. Then $\operatorname{ind}\left(C_{\mathfrak{g}}(x)\right)$ is equal to the rank of $\mathfrak{g}$.

This conjecture has recently received renewed attention, cf. [5], [6], [10]. Its proof immediately reduces to the case where $\mathfrak{g}$ is simple, and $x$ nilpotent (cf. $[\mathbf{6}], \S 3$ ). Also an inequality of Vinberg states that ind $\left(C_{\mathfrak{g}}(x)\right)$ is at least the rank of $\mathfrak{g}$ (see [6], $1.6,1.7)$. The conjecture has been proved for $\mathfrak{g}$ of classical type in $[\mathbf{1 1}]$ (see also the discussion in [5]). In Section 4 we report on computer calculations that settle the conjecture for the exceptional types.

In $[9]$ the question is considered whether for a given nilpotent $e \in \mathfrak{g}$ there exists $x \in C_{\mathfrak{g}}(e)$ such that the dimension of $C_{\mathfrak{g}}(e, x)$ equals the rank of $\mathfrak{g}$. There an example is given where such an $x$ does not exist, for the case where $\mathfrak{g}$ is of type $F_{4}$. In Section 5 we approach this question using our lists of representatives of nilpotent orbits. This way we are able to give a complete list of all $e$ for which such an $x$ does not exist, in all exceptional types. For the Lie algebra of type $E_{8}$ this solves an open problem from $[\mathbf{9}]$. For type $G_{2}$ this corrects a statement in [9].

The paper ends with two appendices. The first contains the lists of representatives of nilpotent orbits. The second (Appendix B) has lists of positive roots as they appear in the computer algebra system GAP. They have been added to help reading the tables of Appendix A.

All algorithms described in this paper have been implemented in the language of the computer algebra system GAP4. The implementations are available from

$$
\text { http://www.lms.ac.uk/jcm/11/lms2007-059/appendix-a }
$$

Acknowledgments. I thank Alexander Elashvili for suggesting all the topics of this paper to me, and for his enthusiastic advice while I was writing it. Also I would like to thank Karin Baur for several helpful email exchanges, and for her comments on earlier versions.

\section{Preliminaries on nilpotent orbits}

In this section we give a short overview of the theory behind the classification of nilpotent orbits. For more detailed accounts we refer to $[\mathbf{2}],[\mathbf{3}]$.

Let $e \in \mathfrak{g}$ be a nilpotent element. Then by the Jacobson-Morozov theorem $e$ lies in a subalgebra of $\mathfrak{g}$ that is isomorphic to $\mathfrak{s l}_{2}$. In other words, there are elements $f, h \in \mathfrak{g}$ with $[e, f]=h,[h, f]=-2 f,[h, e]=2 e$. In this case we say that $(f, h, e)$ is an $\mathfrak{s l}_{2}$-triple.

Now let $(f, h, e)$ be an $\mathfrak{s l}_{2}$-triple. Then by the representation theory of $\mathfrak{s l}_{2}$ we get a direct sum decomposition $\mathfrak{g}=\bigoplus_{k \in \mathbb{Z}} \mathfrak{g}(k)$, where $\mathfrak{g}(k)=\{x \in \mathfrak{g} \mid[h, x]=k x\}$. Fix a Cartan subalgebra $H$ of $\mathfrak{g}$ with $h \in H$. Let $\Phi$ be the corresponding root system of $\mathfrak{g}$. For $\alpha \in \Phi$ we let $x_{\alpha}$ be a corresponding root vector. For each $\alpha$ there is a $k \in \mathbb{Z}$ with $x_{\alpha} \in \mathfrak{g}(k)$. We write $\eta(\alpha)=k$. It can be shown that there exists a basis of simple roots $\Delta \subset \Phi$ such that $\eta(\alpha) \geqslant 0$ for all $\alpha \in \Delta$. Furthermore, for such a $\Delta$ 
we have $\eta(\alpha) \in\{0,1,2\}$ for all $\alpha \in \Delta$. Write $\Delta=\left\{\alpha_{1}, \ldots, \alpha_{l}\right\}$. Then the Dynkin diagram of $\Phi$ has $l$ nodes, the $i$ th node corresponding to $\alpha_{i}$. Now to each node we add the label $\eta\left(\alpha_{i}\right)$; the result is called the weighted Dynkin diagram. It is denoted $\Delta(e)$, and it depends only on $e$, and not on the choice of $\mathfrak{s l}_{2}$-triple containing $e$.

Let $e, e^{\prime}$ be two nilpotent elements in $\mathfrak{g}$. It can be shown that $e, e^{\prime}$ lie in the same $G$-orbit if and only if $\Delta(e)=\Delta\left(e^{\prime}\right)$. So the weighted Dynkin diagram of $e$ uniquely identifies the nilpotent orbit $G e$. The weighted Dynkin diagrams corresponding to nilpotent orbits have been classified. For the exceptional types there are explicit lists. For the classical types there is a classification in terms of partitions. In particular, the nilpotent orbits in $\mathfrak{g}$ have been classified.

Let $e \in \mathfrak{g}$ be a representative of a nilpotent orbit. We may assume that $e$ is a linear combination of root vectors, corresponding to positive roots. Let $\beta_{1}, \ldots, \beta_{r}$ be the positive roots involved in this linear combination. Let $x_{\beta_{i}}$ (respectively $y_{\beta_{i}}$ ) be the root vector corresponding to $\beta_{i}$ (respectively $-\beta_{i}$ ). Let $\mathfrak{l} \subset \mathfrak{g}$ be the subalgebra generated by $H$ along with the $x_{\beta_{i}}$ and $y_{\beta_{i}}$. Then $\mathfrak{l}$ is reductive, and $e \in \mathfrak{l}$. Let $(f, h, e)$ be an $\mathfrak{s l}_{2}$-triple containing $e$, contained in $\mathfrak{l}$. Then $\mathfrak{l}$ decomposes with respect to the action of ad $h$ as $\mathfrak{l}=\bigoplus_{k \in \mathbb{Z}} \mathfrak{l}(k)$. Let $\mathfrak{p}=\bigoplus_{k \geqslant 0} \mathfrak{l}(k)$, which is a subalgebra of $\mathfrak{l}$. Now it can be shown that the nilpotent orbit containing $e$ is uniquely determined by the pair (l, p) (cf. [3], Chapter 8). Corresponding to this the nilpotent orbit has the label $X_{n}\left(a_{i}\right)$, where $X_{n}$ is the type of the semisimple part of $\mathfrak{l}$, and $i$ is the number of simple roots in the semisimple part of $\mathfrak{p}$. If the latter algebra is solvable, then we omit the $a_{i}$. Furthermore, if the roots of $\mathfrak{l}$ are short (seen as roots of $\mathfrak{g}$ ), then a tilde is put over the $X_{n}$. On some occasions, two different orbits can have the same label. Then a ' is added to one of them, whereas the other gets ". We note that, although the pair $(\mathfrak{l}, \mathfrak{p})$ uniquely determines the nilpotent orbit, it is also true that the same nilpotent orbit can have more than one (non-isomorphic) such pair. So the same nilpotent orbit can have more than one label.

The nilpotent element $e$ from above also has a Dynkin diagram, which is simply the Dynkin diagram of the roots $\beta_{i}$. This diagram has $r$ nodes, and node $i$ is connected to node $j$ by $\left\langle\beta_{i}, \beta_{j}^{\vee}\right\rangle\left\langle\beta_{j}, \beta_{i}^{\vee}\right\rangle=0,1,2,3$ lines. Furthermore, if these scalar products are positive, then the lines are dotted. This only occurs when $\mathfrak{p}$ is not solvable.

\section{Finding representatives of nilpotent orbits}

In this section we consider the problem of finding a nilpotent element in $\mathfrak{g}$ corresponding to a given weighted Dynkin diagram $D$. We write $D_{i}$ for the label at node $i$. Let $H$ be a fixed Cartan subalgebra of $\mathfrak{g}$.

Let $e \in \mathfrak{g}$ be a nilpotent element such that $\Delta(e)=D$. Then there is an $\mathfrak{s l}_{2}$-triple $(f, h, e)$, containing $e$. Since we can conjugate any Cartan subalgebra of $\mathfrak{g}$ to $H$ by an element of $G$, we may assume that $h \in H$. As in the previous section we write $\Delta=\left\{\alpha_{1}, \ldots, \alpha_{l}\right\}$ for a basis of simple roots. By choosing a Chevalley basis in $\mathfrak{g}$ we get basis elements $h_{1}, \ldots, h_{l}$ of $H$, and root vectors $x_{\alpha_{i}}$ with $\left[h_{j}, x_{\alpha_{i}}\right]=\left\langle\alpha_{i}, \alpha_{j}^{\vee}\right\rangle x_{\alpha_{i}}$.

Each $h \in H$ yields a decomposition $\mathfrak{g}=\bigoplus_{k \in \mathbb{Z}} \mathfrak{g}(k)$, and a weighted Dynkin diagram, as described in the previous section. This weighted Dynkin diagram is equal to $D$ if and only if $\left[h, x_{\alpha_{i}}\right]=D_{i} x_{\alpha_{i}}$ for $1 \leqslant i \leqslant l$. But this happens if and only if $\sum_{j=1}^{l}\left\langle\alpha_{i}, \alpha_{j}^{\vee}\right\rangle a_{j}=D_{i}$, where the $a_{j}$ are such that $h=\sum_{j} a_{j} h_{j}$. Let $C=$ $\left(\left\langle\alpha_{i}, \alpha_{j}^{\vee}\right\rangle\right)_{1 \leqslant i, j \leqslant l}$ be the Cartan matrix of $\Phi$. It follows that $h$ yields the weighted 
Dynkin diagram $D$ if and only if $C\left(a_{1}, \ldots, a_{l}\right)^{t}=\left(D_{1}, \ldots, D_{l}\right)$. Hence there is a unique such $h$, and we can compute it by solving a system of linear equations. However, not every weighted Dynkin diagram corresponds to a nilpotent orbit. In other words, not every weighted Dynkin diagram yields a $h$ that lies in an $\mathfrak{s l}_{2}$-triple. The next two lemmas lead to a probabilistic algorithm to decide whether this is the case or not.

Lemma 1. Let $h \in H$. Then $h$ belongs to an $\mathfrak{s l}_{2}$-triple if and only if there is an $x \in \mathfrak{g}(2)$ such that $h \in[x, \mathfrak{g}(-2)]$.

Proof. The condition is clearly necessary. If $h \in[x, \mathfrak{g}(-2)]$ then there is a $y \in \mathfrak{g}(-2)$ with $[x, y]=h$. Then $(y, h, x)$ is an $\mathfrak{s l}_{2}$-triple.

Lemma 2. Let $h \in H$ be contained in an $\mathfrak{s l}_{2}$-triple $(y, h, x)$. Let $E$ be the set of $x^{\prime} \in \mathfrak{g}(2)$ such that $h \in\left[x^{\prime}, \mathfrak{g}(-2)\right]$. Then $E$ is Zariski dense in $\mathfrak{g}(2)$.

Proof. (cf. [2], Proposition 5.6.2). Let $G_{h}=\{g \in G \mid \operatorname{Ad}(g)(h)=h\}$ be the stabilizer of $h$ in $G$. Then $G_{h}$ is an algebraic subgroup of $G$. Now $\operatorname{Lie}\left(G_{h}\right)=\{u \in$ $\mathfrak{g} \mid \operatorname{ad}(u)(h)=0\}$. This is the centralizer of $h$ in $\mathfrak{g}$. Hence $\operatorname{Lie}\left(G_{h}\right)=\mathfrak{g}(0)$. For $u \in \mathfrak{g}(2)$ and $g \in G_{h}$ we have $[h, \operatorname{Ad}(g)(u)]=\operatorname{Ad}(g)\left[\operatorname{Ad}\left(g^{-1}\right)(h), u\right]=\operatorname{Ad}(g)[h, u]=$ $2 \operatorname{Ad}(g)(u)$. Hence $\operatorname{Ad}(g)$ stabilizes $\mathfrak{g}(2)$. Let $\varphi: G_{h} \rightarrow \mathfrak{g}(2)$ be the morphism defined by $\varphi(g)=\operatorname{Ad}(g)(x)$. Then the image of $\varphi$ is the $G_{h}$-orbit of $x$ in $\mathfrak{g}(2)$. The differential of $\varphi$ is $d \varphi: \mathfrak{g}(0) \rightarrow \mathfrak{g}(2), d \varphi(u)=[u, x]$. But this is surjective because $[\mathfrak{g}(0), x]=\mathfrak{g}(2)$ (this follows from the representation theory of $\mathfrak{s l}_{2}$ ). So $\varphi$ is a dominant morphism. Hence $\varphi\left(G_{h}\right)$ is a dense subset of $\mathfrak{g}(2)$. Furthermore $\varphi\left(G_{h}\right) \subset E$.

Based on this we have a probabilistic algorithm for finding a representative of a nilpotent orbit, given a weighted Dynkin diagram. First we determine the unique $h \in H$ corresponding to the diagram. Then we select a random $x \in \mathfrak{g}(2)$, in the following way. Let $x_{1}, \ldots, x_{s}$ be a basis of $\mathfrak{g}(2)$. Let $\Omega$ be a finite subset of $\mathbb{Q}$ and select $\mu_{1}, \ldots, \mu_{s}$ randomly, uniformly and independently from $\Omega$. Then set $x=\sum_{i} \mu_{i} x_{i}$. By the previous lemma the probability that $h \in[x, \mathfrak{g}(-2)]$ is high (and can be made arbitrarily close to 1 by enlarging $\Omega$ ). If it happens to be the case that $h \notin[x, \mathfrak{g}(-2)]$ then we select another $x$ and continue. This algorithm will terminate in very few steps.

The $x$ found by the algorithm above will have "ugly" coefficients with respect to a Chevalley basis. We can obtain an element with "nice" coefficients in the following way. We write $x$ with respect to a Chevalley basis of $\mathfrak{g}$. We fix every coefficient but the first. For the first coefficient we try the values $0,1,2, \ldots$. The lemma ensures that we will quickly find an $x^{\prime}$ which is a representative of the same nilpotent orbit, with the first coefficient a nice integer. We continue this way until all coefficients are nice integers.

The above results also provide a probabilistic algorithm for testing whether a given weighted Dynkin diagram corresponds to a nilpotent orbit. We basically try the same algorithm a few times, and if it does not come up with an $x$ then the weighted Dynkin diagram does not correspond to a nilpotent orbit with high probability. In principle we can make this absolutely sure by using Gröbner bases. This works as follows. Let $x_{1}, \ldots, x_{s}$ and $y_{1}, \ldots, y_{s}$ be bases of respectively $\mathfrak{g}(2)$ and $\mathfrak{g}(-2)$. Let $a_{1}, \ldots, a_{s}, b_{1}, \ldots, b_{s}$ be indeterminates. Let $u_{1}, \ldots, u_{r}$ be a basis of $\mathfrak{g}(0)$, 
and write $\left[x_{i}, y_{j}\right]=\sum_{k} \gamma_{i j}^{k} u_{k}$, and $h=\sum_{k} \alpha_{k} u_{k}$. Then there is an $x \in \mathfrak{g}(2)$ with $h \in[x, \mathfrak{g}(-2)]$ if and only if the system of polynomial equations

$$
\sum_{i=1}^{s} \sum_{j=1}^{s} \gamma_{i j}^{k} a_{i} b_{j}-\alpha_{k}=0 \quad \text { for } 1 \leqslant k \leqslant r
$$

has a solution. Now this system has a solution over $\mathbb{C}$ if and only if the reduced Gröbner basis of the ideal generated by the left hand sides of these equations is not $\{1\}$.

Remark. In [7] Popov has given an algorithm for determining the strata of the nullcone of a linear representation of a reductive algebraic group. This also yields an algorithm for classifying nilpotent orbits in reductive Lie algebras, and for finding representatives of them.

\section{Calculating the index}

In this section we describe a simple algorithm that for a Lie algebra gives an upper bound for its index. If the Lie algebra is defined over a sufficiently large field (e.g., of characteristic 0), then the probability that this upper bound is equal to the index can be made arbitrarily high. We use the same notation as in Section 1.

Let $K$ be a finite-dimensional Lie algebra with basis $\left\{x_{1}, \ldots, x_{n}\right\}$. Let $c_{i j}^{k}$ be the structure constants of $K$, i.e., $\left[x_{i}, x_{j}\right]=\sum_{k=1}^{n} c_{i j}^{k} x_{k}$. Let $\left\{\psi_{1}, \ldots, \psi_{n}\right\}$ be the dual basis of $K^{*}$, i.e., $\psi_{i}\left(x_{j}\right)=\delta_{i j}$. Let $f=\sum_{i} T_{i} \psi_{i}$ be an element of the dual space $K^{*}$. Let $x=\sum_{i} \alpha_{i} x_{i} \in K$. Then $x \in K^{f}$ if and only if $f\left(\left[x, x_{j}\right]\right)=0$ for $1 \leqslant j \leqslant n$. Now this is equivalent to

$$
\sum_{i=1}^{n}\left(\sum_{k=1}^{n} c_{i j}^{k} T_{k}\right) \alpha_{i}=0 \text { for } j=1, \ldots, n .
$$

Define the $n \times n$-matrix $A$ by $A(i, j)=\sum_{k=1}^{n} c_{i j}^{k} T_{k}$. Then $\operatorname{dim} K^{f}=n-\operatorname{rank}(A)$. So the dimension of $K^{f}$ is minimal if and only if the rank of $A$ is maximal. Now the rank of $A$ is not maximal if and only if certain polynomial expressions in the $T_{k}$ (i.e., determinants of certain minors of $A$ ) vanish. Therefore, if the $T_{k}$ are chosen randomly and uniformly from a sufficiently large set, then with high probability the rank of $A$ will be maximal.

Here we consider the case where $K=C_{\mathfrak{g}}(e)$, where $e$ is a nilpotent element of the simple Lie algebra $\mathfrak{g}$. Then by Vinberg's inequality we have that $\operatorname{ind}(K)$ is at least the rank of $\mathfrak{g}$. So if we find an $f \operatorname{such}$ that $\operatorname{dim}\left(K^{f}\right)=\operatorname{rank}(\mathfrak{g})$, then we have proved that $\operatorname{ind}(K)=\operatorname{rank}(\mathfrak{g})$. Moreover, the above discussion shows that we will quickly find such an $f$ (if it exists) by randomly choosing the $T_{k}$.

With the help of an implementation of this algorithm in GAP4, we have checked Elashvili's conjecture for the exceptional types (which, except $G_{2}$, are the remaining open cases). As a result we can conclude that Elashvili's conjecture holds for all simple Lie algebras. 


\section{Centralizers in centralizers}

Let $e \in \mathfrak{g}$ be a nilpotent element. Let $C_{e}=C_{\mathfrak{g}}(e)$ be the centralizer of $e$ in $\mathfrak{g}$. Let $x \in C_{e}$ and consider the centralizer $C_{e, x}$ of $x$ in $C_{e}$ (i.e., $C_{e, x}$ is the set of all elements of $\mathfrak{g}$ commuting with both $e$ and $x$ ). From $[\mathbf{8}]$ it follows that $C_{e, x}$ contains a commutative subalgebra of dimension equal to $\operatorname{rank}(\mathfrak{g})$. Hence the dimension of $C_{e, x}$ is at least the rank of $\mathfrak{g}$. In $[\mathbf{9}]$ the following question is considered: given $e$ does there exist $x \in C_{e}$ such that the dimension of $C_{e, x}$ equals the rank of $\mathfrak{g}$ ? The main result of that paper is a counter example to the question for the case where $\mathfrak{g}$ is of type $F_{4}$.

With the lists of representatives of the nilpotent orbits we can easily tackle this question in all simple Lie algebras of exceptional type. Let $e \in \mathfrak{g}$ be a nilpotent element, and let $x_{1}, \ldots, x_{m}$ be a basis of $C_{e}$. Set $x=T_{1} x_{1}+\cdots+T_{m} x_{m}$. Then the centralizer of $x$ in $C_{e}$ is equal to the kernel of ad $x$ (restricted to $C_{e}$ ). So the dimension of $C_{e, x}$ is minimal if the rank of the matrix adx is maximal. Now the entries of this matrix are linear polynomials in the $T_{i}$. It follows that for a random choice of the $T_{i}$, with very high probability, the $\operatorname{rank}$ of $\operatorname{ad} x$ is $\operatorname{maximal}$. So this gives a probabilistic algorithm for determining the minimal dimension of $C_{e, x}$ (recall that we are varying $x$, and keeping $e$ fixed). Once the minimal dimension is found with this algorithm we can prove it rigorously as follows. Let $x$ be an element such that $\operatorname{dim} C_{e, x}$ is (hypothetically) minimal, as produced by the algorithm. If $\operatorname{dim} C_{e, x}=\operatorname{rank}(\mathfrak{g})$ then we have proved that the minimal dimension of a $C_{e, x}$ is $\operatorname{rank}(\mathfrak{g})$, as it cannot be smaller. Secondly, if the dimension that we find happens to be bigger, then we compute the rank of the matrix ad $x$, where $x=T_{1} x_{1}+\cdots+T_{m} x_{m}$ and we let the $T_{i}$ be generators of a rational function field. The rank of that matrix will equal the maximal rank of any ad $x$ for $x \in C_{e}$.

Using this algorithm we arrive at the following result.

Proposition 1. Let $\mathfrak{g}$ be a simple Lie algebra of exceptional type, and $e \in \mathfrak{g}$ nilpotent. Then the minimal dimension of $a C_{e, x}$ is equal to $\operatorname{rank}(\mathfrak{g})$, except in three cases, which are listed in the following table:

\begin{tabular}{|l|l|l|}
\hline type of $\mathfrak{g}$ & label of $e$ & dimension of minimal $C_{e, x}$ \\
\hline$G_{2}$ & $A_{1}+\widetilde{A}_{1}$ & 3 \\
$F_{4}$ & $\widetilde{A_{2}}+A_{2}$ & 6 \\
$E_{8}$ & $A_{5}+A_{2}+A_{1}$ & 12 \\
\hline
\end{tabular}

In all three cases it turns out that a minimal $C_{e, x}$ is abelian. Furthermore, in each case it is possible to choose the element $x \in C_{e}$ such that it is homogeneous of degree -1 with respect to the grading of $\mathfrak{g}$ defined by the $\mathfrak{s l}_{2}$-triple containing e.

In relation to $[\mathbf{9}]$ we remark the following. In $[\mathbf{9}]$ it is wrongly stated that in $G_{2}$ all minimal $C_{e, x}$ have dimension equal to $\operatorname{rank}(\mathfrak{g})$. The result for $F_{4}$ is the same as in $[\mathbf{9}]$. Finally, the problem for $E_{8}$ is left open in $[\mathbf{9}]$.

Also, as a straightforward corollary of the proposition, it follows that in the exceptional types a minimal $C_{e, x}$ is always abelian. 


\section{Appendix A. Representatives of nilpotent orbits}

In the tables below we list the nilpotent orbits in the Lie algebras of exceptional type. For each orbit we have given a label, the weighted Dynkin diagram, and the Dynkin diagram of a representative. We remark the following. If more than one label was possible, we have chosen the simplest one that we could find. This means that we have preferred a label of the form $X_{n}$ over a label of the form $X_{n}\left(a_{i}\right)$. Furthermore, we have preferred labels such that the Dynkin diagram of a corresponding representative has as few lines as possible. In the Dynkin diagram a black node means that the corresponding root is long. Finally, the labels corresponding to each node refer to the basis elements of the simple Lie algebras as present in GAP4. In Appendix B we list the positive roots of each root system of exceptional type, in the order in which they are used by GAP4. Now, if in the tables in this section a Dynkin diagram of a representative has labels $i_{1}, \ldots, i_{k}$, then the corresponding representative is the sum of the root vectors corresponding to the $i_{j}$ th positive root for $1 \leqslant j \leqslant k$.

Table 2: Nilpotent orbits in the Lie algebra of type $G_{2}$.

\begin{tabular}{|c|c|c|}
\hline label & diagram & representative \\
\hline$A_{1}$ & 10 & $e^{6}$ \\
\hline$\widetilde{A}_{1}$ & $\begin{array}{ll}0 & 1\end{array}$ & $0^{4}$ \\
\hline$A_{1}+\widetilde{A}_{1}$ & 20 & $\bullet^{2} 0^{4}$ \\
\hline$G_{2}$ & $2 \quad 2$ & $\stackrel{(=}{=}^{2}$ \\
\hline
\end{tabular}

Table 3: Nilpotent orbits in the Lie algebra of type $F_{4}$.

\begin{tabular}{|c|c|c|c|c|c|}
\hline label & & liag & $\mathrm{ran}$ & & representative \\
\hline$A_{1}$ & 1 & 0 & 0 & & $\bullet^{24}$ \\
\hline$\widetilde{A}_{1}$ & 0 & 0 & 0 & 1 & $\mathrm{O}^{21}$ \\
\hline$A_{1}+\widetilde{A}_{1}$ & 0 & 1 & 0 & 0 & $0^{17} \bullet^{22}$ \\
\hline$A_{2}$ & 2 & 0 & 0 & 0 & $\overbrace{}^{16}$ \\
\hline$\widetilde{A}_{2}$ & 0 & 0 & 0 & 2 & $\mathrm{O}^{11} \mathrm{O}^{12}$ \\
\hline$\widetilde{A}_{1}+A_{2}$ & 0 & 0 & 1 & 0 & $0^{14} \bullet^{15}$ \\
\hline$B_{2}$ & 2 & 0 & 0 & 1 & $\stackrel{9}{\rightleftharpoons}$ \\
\hline$\widetilde{A}_{2}+A_{1}$ & 0 & 1 & 0 & 1 & $\mathrm{O}^{8} \mathrm{O}^{14} \bullet^{16}$ \\
\hline$B_{2}+A_{1}$ & 1 & 0 & 1 & 0 & $\stackrel{10}{=}^{11} \bullet^{15}$ \\
\hline$\widetilde{A}_{2}+A_{2}$ & 0 & 2 & 0 & 0 & $\overbrace{}^{8} \overbrace{}^{9} \overbrace{}^{18}$ \\
\hline
\end{tabular}


Computing with nilpotent orbits in simple Lie algebras of exceptional type

\begin{tabular}{|c|c|c|c|c|c|}
\hline$B_{3}$ & 2 & 2 & 0 & 0 & $\stackrel{8}{=} \overbrace{}^{10}$ \\
\hline$C_{3}$ & 1 & 0 & 1 & 2 & $\stackrel{10}{=} \mathrm{O}^{1}$ \\
\hline$C_{3}+A_{1}$ & 0 & 2 & 0 & 2 & $\stackrel{6}{=} 0^{5}-0^{7} \bullet^{13}$ \\
\hline$B_{4}$ & 2 & 2 & 0 & 2 & $\stackrel{5}{\overbrace{}^{4}} \overbrace{}^{10}$ \\
\hline$F_{4}$ & 2 & 2 & 2 & 2 & $\mathrm{O}^{1} \overbrace{}^{3}$ \\
\hline
\end{tabular}

Table 4: Nilpotent orbits in the Lie algebra of type $E_{6}$.

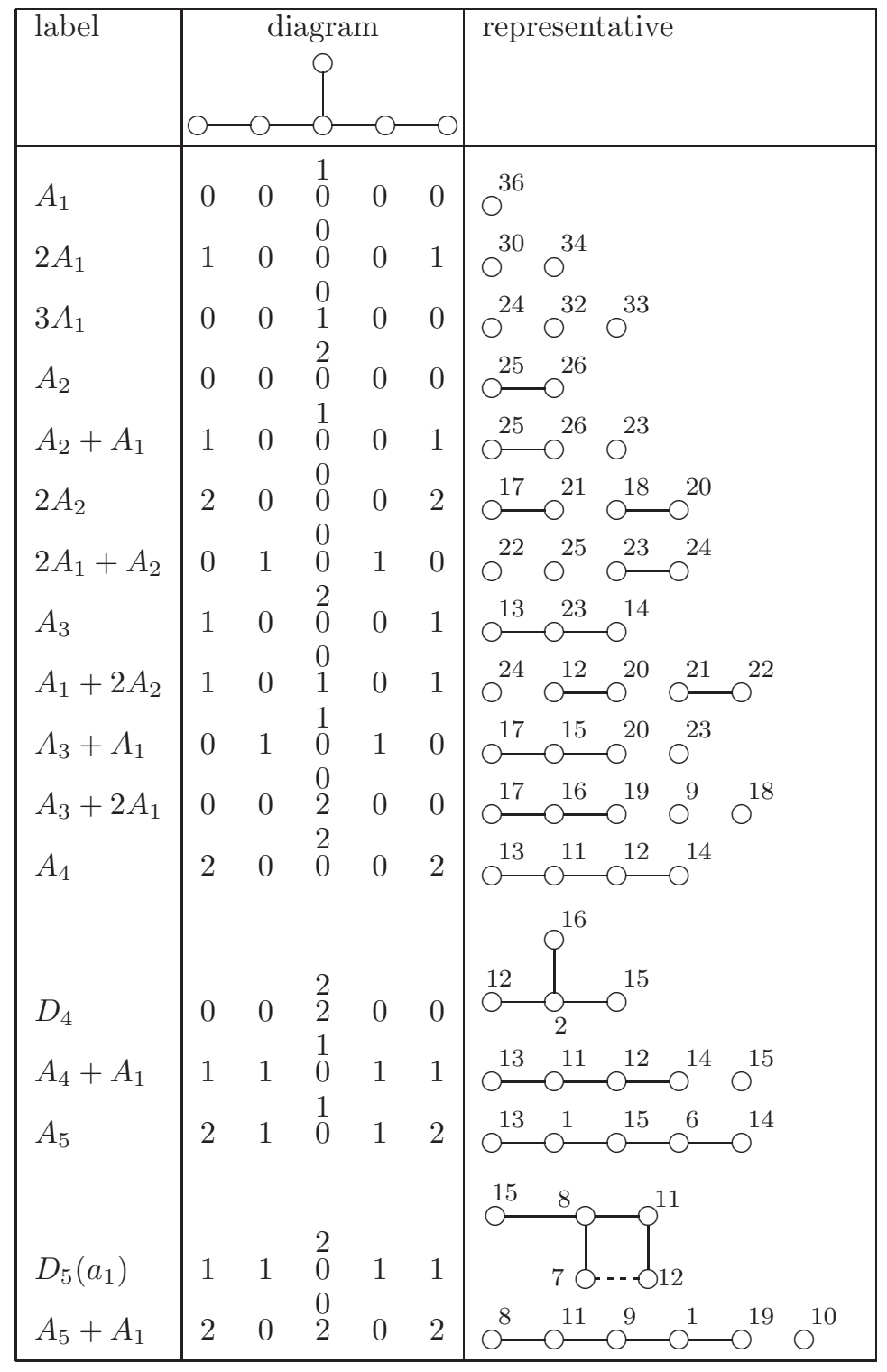




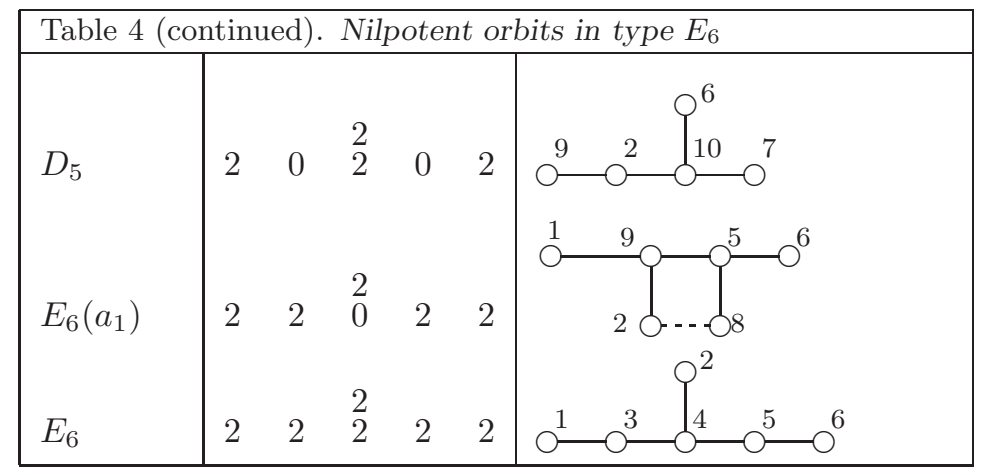

Table 5: Nilpotent orbits in the Lie algebra of type $E_{7}$.

\begin{tabular}{|c|c|c|c|c|c|c|c|}
\hline label & \multicolumn{6}{|c|}{ diagram } & representative \\
\hline$A_{1}$ & 1 & 0 & $\begin{array}{l}0 \\
0\end{array}$ & 0 & 0 & 0 & $0^{63}$ \\
\hline $2 A_{1}$ & 0 & 0 & $\begin{array}{l}0 \\
0\end{array}$ & 0 & 1 & 0 & $0^{57} \bigcirc^{60}$ \\
\hline$\left(3 A_{1}\right)^{\prime \prime}$ & 0 & 0 & $\begin{array}{l}0 \\
0\end{array}$ & 0 & 0 & 2 & $\bigcirc^{47} \bigcirc^{48} \bigcirc^{49}$ \\
\hline$\left(3 A_{1}\right)^{\prime}$ & 0 & 1 & $\begin{array}{l}0 \\
0\end{array}$ & 0 & 0 & 0 & $\bigcirc^{42} \bigcirc^{56} \bigcirc^{59}$ \\
\hline$A_{2}$ & 2 & 0 & $\begin{array}{l}0 \\
0\end{array}$ & 0 & 0 & 0 & $\mathrm{O}^{44}$ \\
\hline $4 A_{1}$ & 0 & 0 & $\begin{array}{l}1 \\
0\end{array}$ & 0 & 0 & 1 & $\bigcirc^{45} \bigcirc^{47} \bigcirc^{52} \bigcirc^{53}$ \\
\hline$A_{2}+A_{1}$ & 1 & 0 & $\begin{array}{l}0 \\
0\end{array}$ & 0 & 1 & 0 & $\stackrel{44}{\mathrm{O}^{46}} \mathrm{O}^{49}$ \\
\hline$A_{2}+2 A_{1}$ & 0 & 0 & $\begin{array}{l}0 \\
1\end{array}$ & 0 & 0 & 0 & $\mathrm{O}^{41} \mathrm{O}^{43} \mathrm{O}^{42} \mathrm{O}^{51}$ \\
\hline$A_{3}$ & 2 & 0 & $\begin{array}{l}0 \\
0\end{array}$ & 0 & 1 & 0 & $\mathrm{O}^{20} \mathrm{O}^{49}$ \\
\hline $2 A_{2}$ & 0 & 0 & $\begin{array}{l}0 \\
0\end{array}$ & 0 & 2 & 0 & $\stackrel{34}{\mathrm{O}^{35}} \mathrm{\bigcirc}^{36} \mathrm{\bigcirc}^{43}$ \\
\hline$A_{2}+3 A_{1}$ & 0 & 0 & $\begin{array}{l}2 \\
0\end{array}$ & 0 & 0 & 0 & $\mathrm{\bigcirc}^{39} \mathrm{\bigcirc}^{40} \mathrm{\bigcirc}^{37} \mathrm{\bigcirc}^{38} \mathrm{\bigcirc}^{41}$ \\
\hline$\left(A_{3}+A_{1}\right)^{\prime \prime}$ & 2 & 0 & $\begin{array}{l}0 \\
0\end{array}$ & 0 & 0 & 2 & $\mathrm{O}^{27} \mathrm{O}^{30} \mathrm{O}^{37} \mathrm{O}^{31}$ \\
\hline $2 A_{2}+A_{1}$ & 0 & 1 & $\begin{array}{l}0 \\
0\end{array}$ & 0 & 1 & 0 & $\mathrm{O}^{34} \mathrm{O}^{35} \mathrm{O}^{36} \mathrm{O}^{43} \mathrm{\bigcirc}^{37}$ \\
\hline$\left(A_{3}+A_{1}\right)^{\prime}$ & 1 & 0 & $\begin{array}{l}0 \\
1\end{array}$ & 0 & 0 & 0 & $\mathrm{O}^{27} \mathrm{O}^{28} \mathrm{O}^{39} \mathrm{O}^{49}$ \\
\hline$\left(A_{3}+2 A_{1}\right)^{\prime}$ & 0 & 2 & $\begin{array}{l}0 \\
0\end{array}$ & 0 & 0 & 0 & $\mathrm{O}^{29} \mathrm{O}^{32} \mathrm{O}^{31} \mathrm{O}^{27} \mathrm{\bigcirc}^{39}$ \\
\hline$\left(A_{3}+2 A_{1}\right)^{\prime \prime}$ & 1 & 0 & $\begin{array}{l}0 \\
0\end{array}$ & 1 & 0 & 1 & $\mathrm{O}^{27} \mathrm{O}^{30} \mathrm{O}^{37} \mathrm{O}^{31} \mathrm{\bigcirc}^{40}$ \\
\hline$D_{4}$ & 2 & 2 & $\begin{array}{l}0 \\
0\end{array}$ & 0 & 0 & 0 & 28 \\
\hline
\end{tabular}




\begin{tabular}{|c|c|c|c|c|c|c|c|}
\hline$A_{3}+3 A_{1}$ & 0 & 1 & $\begin{array}{l}1 \\
0\end{array}$ & 0 & 0 & 1 & $\mathrm{O}^{32} \mathrm{O}^{31} \mathrm{O}^{33} \mathrm{O}^{22} \mathrm{O}^{30} \mathrm{\bigcirc}^{35}$ \\
\hline$A_{3}+A_{2}$ & 0 & 0 & $\begin{array}{l}0 \\
1\end{array}$ & 0 & 1 & 0 & $\mathrm{O}^{29} \mathrm{O}^{32}=\mathrm{O}^{31} \mathrm{O}^{27} \mathrm{O}^{30}$ \\
\hline$A_{4}$ & 2 & 0 & $\begin{array}{l}0 \\
0\end{array}$ & 0 & 2 & 0 & $\mathrm{O}^{21} \mathrm{O}^{29} \mathrm{O}^{25} \mathrm{O}^{20}$ \\
\hline$A_{3}+A_{2}+A_{1}$ & 0 & 0 & $\begin{array}{l}0 \\
0\end{array}$ & 2 & 0 & 0 & $\mathrm{O}^{26} \mathrm{O}^{25} \mathrm{O}^{29} \mathrm{O}^{27} \mathrm{O}^{28} \mathrm{O}^{47}$ \\
\hline$\left(A_{5}\right)^{\prime \prime}$ & 2 & 0 & $\begin{array}{l}0 \\
0\end{array}$ & 0 & 2 & 2 & $\mathrm{O}^{20} \mathrm{O}^{24} \mathrm{O}^{7}=\mathrm{O}^{23} \mathrm{O}^{21}$ \\
\hline$D_{4}+A_{1}$ & 2 & 1 & $\begin{array}{l}1 \\
0\end{array}$ & 0 & 0 & 1 & $\bigcirc_{1}^{28}-\bigcirc^{31} \bigcirc^{30}$ \\
\hline$A_{4}+A_{1}$ & 1 & 0 & $\begin{array}{l}0 \\
1\end{array}$ & 0 & 1 & 0 & $\mathrm{O}^{20} \longrightarrow \mathrm{O}^{25} \stackrel{29}{-} \mathrm{O}^{21} \mathrm{O}^{28}$ \\
\hline$D_{4}+2 A_{1}$ & 2 & 0 & $\begin{array}{l}0 \\
1\end{array}$ & 0 & 1 & 0 & $\bigcirc_{8}^{18}-\mathrm{O}^{28} \mathrm{O}^{29} \mathrm{O}^{31}$ \\
\hline$A_{4}+A_{2}$ & 0 & 0 & 0 & 0 & 0 & 0 & $\mathrm{O}^{22} \mathrm{O}^{25} \mathrm{O}^{20}=\mathrm{O}^{24} \mathrm{O}^{21} \mathrm{O}^{23}$ \\
\hline$\left(A_{5}\right)^{\prime}$ & 1 & 0 & $\begin{array}{l}0 \\
1\end{array}$ & 0 & 2 & 0 & $\mathrm{O}^{20} \mathrm{O}^{12} \mathrm{O}^{28}=\mathrm{O}^{13}$ \\
\hline$\left(A_{5}+A_{1}\right)^{\prime \prime}$ & 1 & 0 & $\begin{array}{l}0 \\
1\end{array}$ & 0 & 1 & 2 & 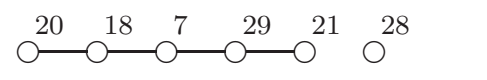 \\
\hline$D_{5}\left(a_{1}\right)+A_{1}$ & 2 & 0 & $\begin{array}{l}0 \\
0\end{array}$ & 2 & 0 & 0 & $0 \quad \bigcirc^{29}$ \\
\hline$D_{6}\left(a_{2}\right)$ & 0 & 1 & $\begin{array}{l}1 \\
0\end{array}$ & 1 & 0 & 2 & $230-16$ \\
\hline$\left(A_{5}+A_{1}\right)^{\prime}$ & 0 & 2 & $\begin{array}{l}0 \\
0\end{array}$ & 0 & 2 & 0 & 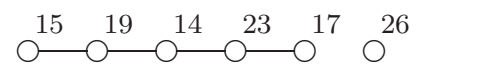 \\
\hline$D_{5}$ & 2 & 2 & $\begin{array}{l}0 \\
0\end{array}$ & 0 & 2 & 0 & $17 \mathrm{O}^{11} \mathrm{C}^{115}$ \\
\hline$A_{5}+A_{2}$ & 0 & 0 & $\begin{array}{l}0 \\
2 \\
0\end{array}$ & 0 & 0 & 2 & $\mathrm{O}^{16} \mathrm{O}^{14} \mathrm{O}^{19}$ \\
\hline$A_{6}$ & 0 & 0 & $\begin{array}{l}0 \\
2\end{array}$ & 0 & 2 & 0 & $\mathrm{O}^{15} \mathrm{O}^{12} \mathrm{O}^{14} \mathrm{O}^{16} \mathrm{C}^{13}$ \\
\hline$D_{5}+A_{1}$ & 2 & 1 & $\begin{array}{l}1 \\
0\end{array}$ & 1 & 0 & 2 & $\mathrm{O}^{17} \mathrm{O}^{1}=\mathrm{O}^{15} \mathrm{O}^{16}$ \\
\hline$D_{6}\left(a_{1}\right)$ & 2 & 1 & $\begin{array}{l}1 \\
0\end{array}$ & 1 & 0 & 2 & 7 \\
\hline
\end{tabular}


Computing with nilpotent orbits in simple Lie algebras of exceptional type

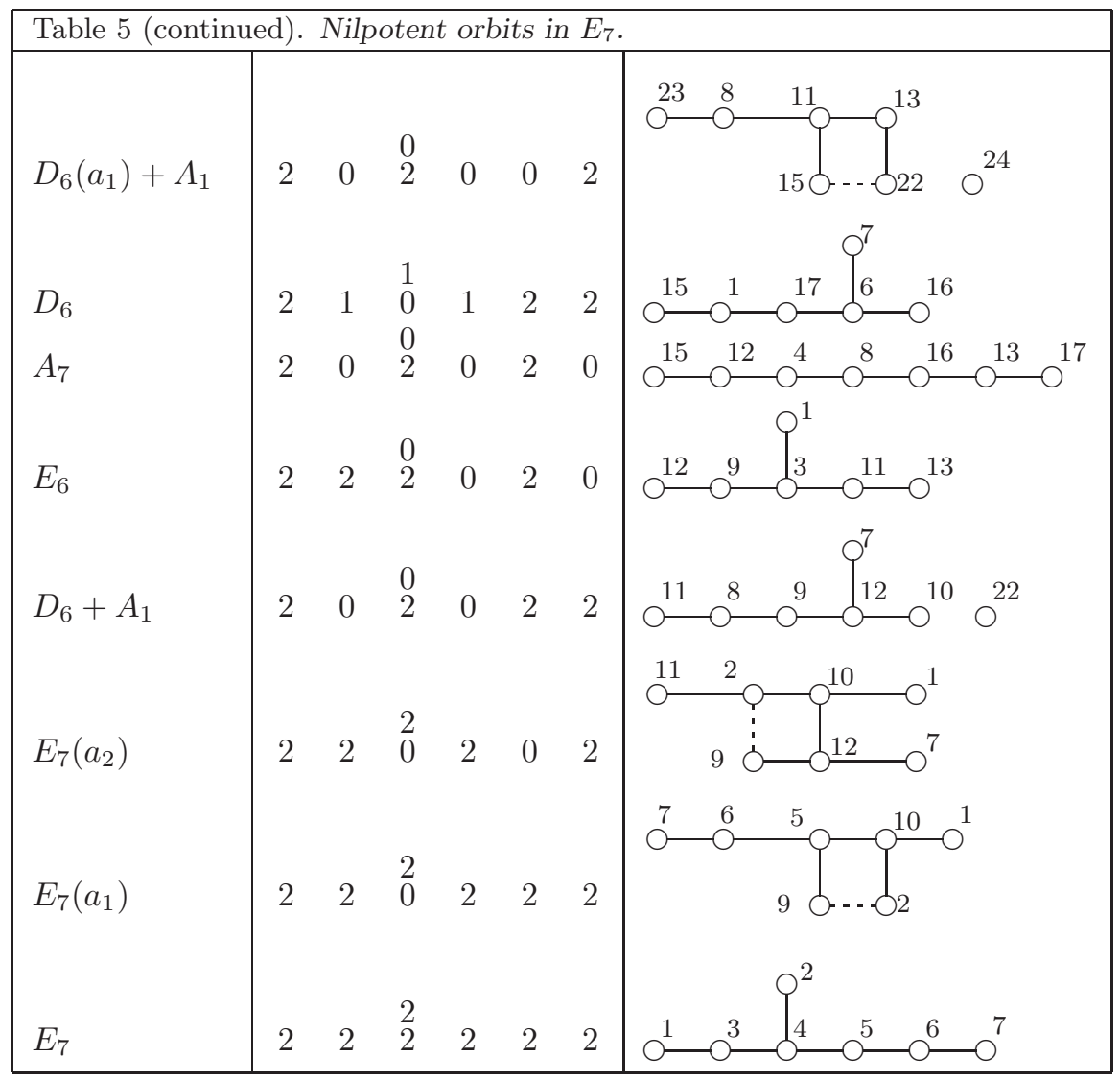

Table 6: Nilpotent orbits in the Lie algebra of type $E_{8}$.

\begin{tabular}{|c|c|c|c|c|c|c|c|c|}
\hline label & \multicolumn{7}{|c|}{ diagram } & representative \\
\hline$A_{1}$ & 0 & 0 & $\begin{array}{l}0 \\
0\end{array}$ & 0 & 0 & 0 & 1 & $0^{120}$ \\
\hline $2 A_{1}$ & 1 & 0 & $\begin{array}{l}0 \\
0\end{array}$ & 0 & 0 & 0 & 0 & $\bigcirc^{113} \bigcirc^{114}$ \\
\hline $3 A_{1}$ & 0 & 0 & $\begin{array}{l}0 \\
0\end{array}$ & 0 & 0 & 1 & 0 & $\bigcirc^{104} \bigcirc^{105} \bigcirc^{106}$ \\
\hline$A_{2}$ & 0 & 0 & $\begin{array}{l}0 \\
0\end{array}$ & 0 & 0 & 0 & 2 & $\mathrm{O}^{88} \mathrm{O}^{90}$ \\
\hline $4 A_{1}$ & 0 & 0 & $\begin{array}{l}1 \\
0\end{array}$ & 0 & 0 & 0 & 0 & $\bigcirc^{95} \bigcirc^{97} \bigcirc^{98} \bigcirc^{103}$ \\
\hline$A_{2}+A_{1}$ & 1 & 0 & $\begin{array}{l}0 \\
0\end{array}$ & 0 & 0 & 0 & 1 & $\mathrm{O}^{88} \mathrm{O}^{90} \mathrm{O}^{97}$ \\
\hline$A_{2}+2 A_{1}$ & 0 & 0 & $\begin{array}{l}0 \\
0\end{array}$ & 0 & 1 & 0 & 0 & $\mathrm{O}^{83} \mathrm{O}^{85} \mathrm{O}^{91} \mathrm{O}^{92}$ \\
\hline$A_{3}$ & 1 & 0 & $\begin{array}{l}0 \\
0\end{array}$ & 0 & 0 & U & 2 & $\mathrm{O}^{42} \mathrm{C}^{97}$ \\
\hline$A_{2}+3 A_{1}$ & 0 & 1 & $\begin{array}{l}0 \\
0\end{array}$ & 0 & 0 & U & 0 & $\mathrm{O}^{77} \mathrm{O}^{80} \bigcirc^{82} \bigcirc^{84} \bigcirc^{90}$ \\
\hline
\end{tabular}


Computing with nilpotent orbits in simple Lie algebras of exceptional type

\begin{tabular}{|c|c|c|c|c|c|c|c|c|}
\hline \multicolumn{9}{|c|}{ Table 6 (continued). Nilpotent orbits in $E_{8}$. } \\
\hline $2 A_{2}$ & 2 & 0 & $\begin{array}{l}0 \\
0\end{array}$ & 0 & 0 & 0 & 0 & $\mathrm{O}^{70} \mathrm{O}^{73} \mathrm{O}^{71} \mathrm{O}^{81}$ \\
\hline $2 A_{2}+A_{1}$ & 1 & 0 & $\begin{array}{l}0 \\
0\end{array}$ & 0 & 0 & 1 & 0 & $\mathrm{O}^{70} \mathrm{\bigcirc}^{73} \mathrm{\bigcirc}^{72} \mathrm{\bigcirc}^{80} \bigcirc^{74}$ \\
\hline$A_{3}+A_{1}$ & 0 & 0 & $\begin{array}{l}0 \\
0\end{array}$ & 0 & 1 & 0 & 1 & $\mathrm{O}^{62} \mathrm{O}^{66} \mathrm{O}^{65} \mathrm{\bigcirc}^{93}$ \\
\hline$\left(A_{3}+2 A_{1}\right)^{\prime}$ & 0 & 0 & $\begin{array}{l}0 \\
0\end{array}$ & 0 & 0 & 2 & 0 & $\mathrm{O}^{61} \mathrm{O}^{60} \mathrm{\bigcirc}^{64} \mathrm{O}^{62} \mathrm{\bigcirc}^{78}$ \\
\hline$D_{4}$ & 0 & 0 & $\begin{array}{l}0 \\
0\end{array}$ & 0 & 0 & 2 & 2 & $\mathrm{O}^{53} \mathrm{O}^{55}$ \\
\hline $2 A_{2}+2 A_{1}$ & 0 & 0 & $\begin{array}{l}0 \\
0\end{array}$ & 1 & 0 & 0 & 0 & $\mathrm{\bigcirc}^{69} \mathrm{\bigcirc}^{74} \mathrm{\bigcirc}^{70} \mathrm{\bigcirc}^{73} \bigcirc^{71} \bigcirc^{72}$ \\
\hline$\left(A_{3}+2 A_{1}\right)^{\prime \prime}$ & 0 & 1 & $\begin{array}{l}0 \\
0\end{array}$ & 0 & 0 & 0 & 1 & $\mathrm{O}^{67} \mathrm{O}^{58} \mathrm{O}^{68} \mathrm{O}^{69} \mathrm{O}^{82}$ \\
\hline$A_{3}+3 A_{1}$ & 0 & 0 & $\begin{array}{l}1 \\
0\end{array}$ & 0 & 0 & 1 & 0 & $\mathrm{O}^{59} \mathrm{O}^{56} \mathrm{\bigcirc}^{71} \mathrm{\bigcirc}^{69} \bigcirc^{72} \bigcirc^{73}$ \\
\hline$A_{3}+A_{2}$ & 1 & 0 & $\begin{array}{l}0 \\
0\end{array}$ & 0 & 1 & 0 & 0 & $\stackrel{45}{\mathrm{O}} \mathrm{O}^{74} \mathrm{O}^{57} \mathrm{O}^{58} \mathrm{O}^{67}$ \\
\hline$A_{4}$ & 2 & 0 & $\begin{array}{l}0 \\
0\end{array}$ & 0 & 0 & 0 & 2 & $\mathrm{O}^{42} \mathrm{O}^{57} \mathrm{O}^{53} \mathrm{O}^{43}$ \\
\hline$A_{3}+A_{2}+A_{1}$ & 0 & 0 & $\begin{array}{l}0 \\
1\end{array}$ & 0 & 0 & 0 & 0 & $\mathrm{O}^{52} \mathrm{\bigcirc}^{58} \mathrm{O}^{62} \mathrm{\bigcirc}^{60} \mathrm{O}^{61} \mathrm{O}^{78}$ \\
\hline$D_{4}+A_{1}$ & 0 & 0 & $\begin{array}{l}1 \\
0\end{array}$ & 0 & 0 & 1 & 2 & $\mathrm{O}^{55} \mathrm{C}_{0}^{58} \mathrm{O}^{69}$ \\
\hline$A_{3}+A_{2}+2 A_{1}$ & 0 & 0 & $\begin{array}{l}2 \\
0\end{array}$ & 0 & 0 & 0 & 0 & $\mathrm{O}^{57} \mathrm{O}^{56} \mathrm{O}^{59} \mathrm{O}^{54} \mathrm{O}^{61} \mathrm{O}^{45} \mathrm{O}^{58}$ \\
\hline$A_{4}+A_{1}$ & 1 & 0 & $\begin{array}{l}0 \\
0\end{array}$ & 0 & 1 & 0 & 1 & $\mathrm{O}^{42} \mathrm{O}^{57} \mathrm{O}^{53} \mathrm{O}^{43} \mathrm{O}^{61}$ \\
\hline $2 A_{3}$ & 1 & 0 & $\begin{array}{l}0 \\
0\end{array}$ & 1 & 0 & 0 & 0 & $\mathrm{O}^{44} \mathrm{O}^{68} \mathrm{O}^{46} \mathrm{O} \stackrel{45}{\mathrm{~S}} \mathrm{O}^{55} \mathrm{O}^{47}$ \\
\hline$D_{4}+2 A_{1}$ & 1 & 0 & $\begin{array}{l}0 \\
0\end{array}$ & 0 & 1 & 0 & 2 & $\bigcirc_{15}^{45} \overbrace{}^{57} \bigcirc^{58} \bigcirc^{59}$ \\
\hline$A_{4}+2 A_{1}$ & 0 & 0 & $\begin{array}{l}0 \\
1\end{array}$ & 0 & 0 & 0 & 1 & $\mathrm{O}^{47} \mathrm{O}^{49} \mathrm{O}^{52} \mathrm{O}^{50} \mathrm{O}^{51} \mathrm{O}^{64}$ \\
\hline$A_{4}+A_{2}$ & 0 & 0 & $\begin{array}{l}0 \\
0\end{array}$ & 0 & 2 & 0 & 0 & $\mathrm{O}^{46} \mathrm{O}^{48} \mathrm{O}^{47} \mathrm{O}^{49} \mathrm{O}^{50}$ \\
\hline$A_{5}$ & 2 & 0 & $\begin{array}{l}0 \\
0\end{array}$ & 0 & 1 & 0 & 1 & $\mathrm{O}^{42} \mathrm{O}^{24} \mathrm{O}^{61} \mathrm{O}^{23} \mathrm{O}^{43}$ \\
\hline$D_{5}\left(a_{1}\right)+A_{1}$ & 0 & 0 & $\begin{array}{l}0 \\
1 \\
0\end{array}$ & 0 & 0 & 0 & 2 & $590-.553 \bigcirc^{71}$ \\
\hline$A_{4}+A_{2}+A_{1}$ & 0 & 1 & $\begin{array}{l}0 \\
0\end{array}$ & 0 & 1 & 0 & 0 & $\mathrm{O}^{46} \mathrm{O}^{48} \mathrm{O}^{47} \mathrm{O}^{49} \mathrm{O}^{45} \mathrm{O}^{50} \mathrm{O}^{44}$ \\
\hline$D_{4}+A_{2}$ & 0 & 0 & $\begin{array}{l}2 \\
0\end{array}$ & 0 & 0 & 0 & 2 & $\mathrm{O}_{43}^{34} \mathrm{\bigcirc}^{37} \mathrm{\bigcirc}^{40} \mathrm{\bigcirc}^{64}$ \\
\hline
\end{tabular}


Computing with nilpotent orbits in simple Lie algebras of exceptional type

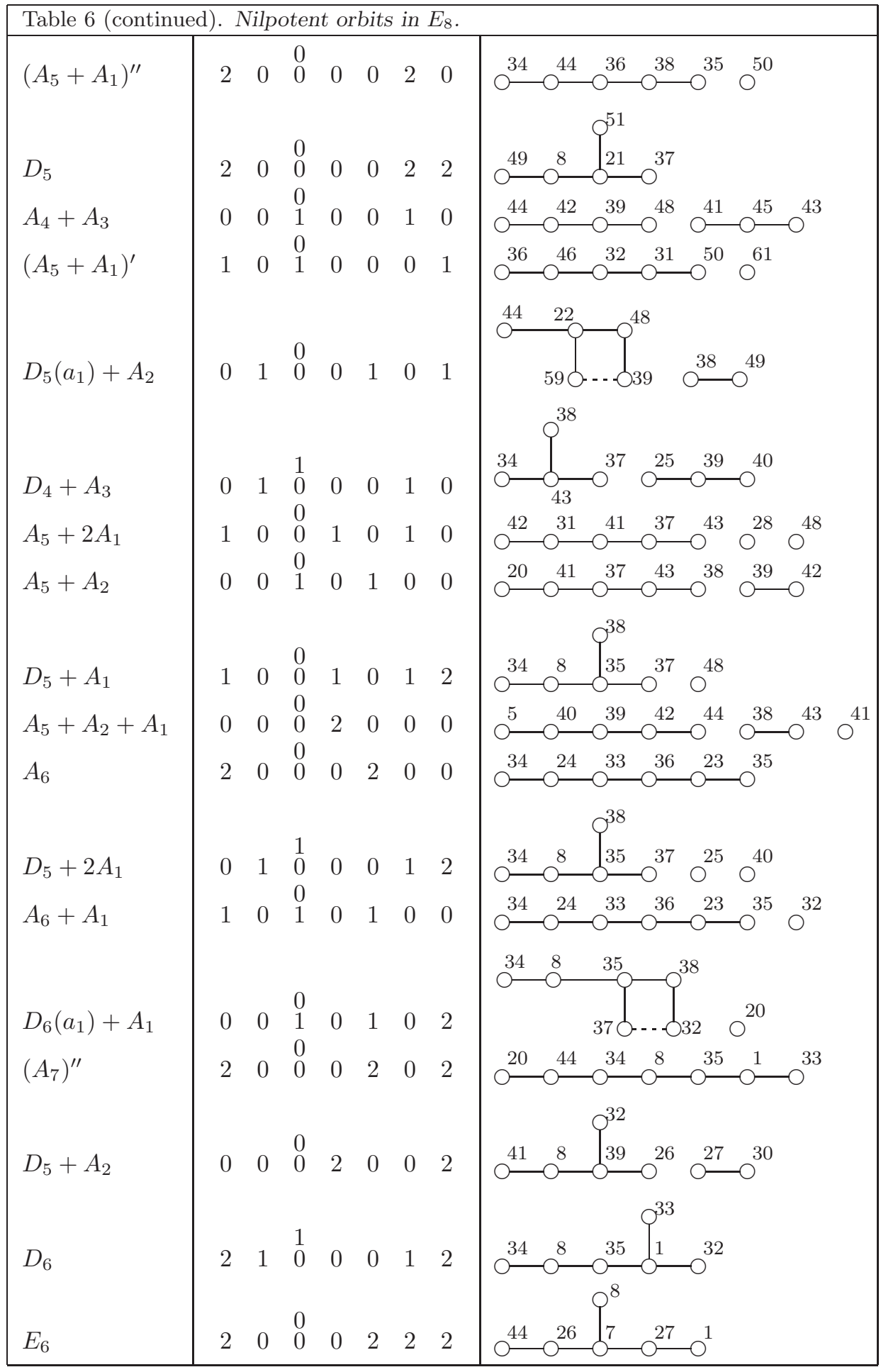


Computing with nilpotent orbits in simple Lie algebras of exceptional type

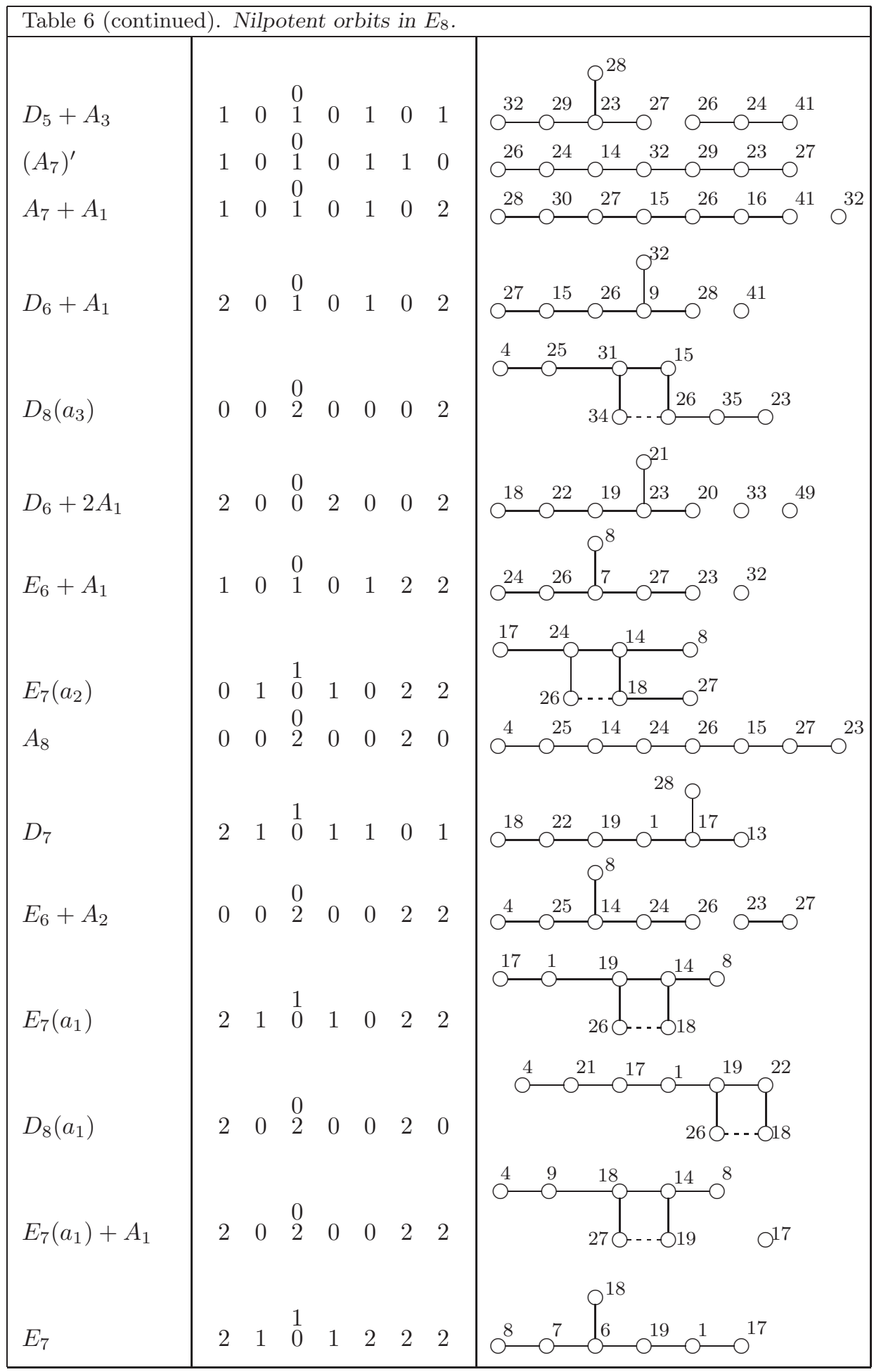


Computing with nilpotent orbits in simple Lie algebras of exceptional type

\begin{tabular}{|c|c|c|c|c|c|c|c|c|}
\hline \multicolumn{9}{|c|}{ Table 6 (continued). Nilpotent orbits in $E_{8}$. } \\
\hline$D_{8}$ & 2 & 0 & $\begin{array}{l}0 \\
2\end{array}$ & 0 & 2 & 0 & 2 & 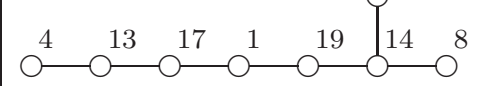 \\
\hline$E_{7}+A_{1}$ & 2 & 0 & $\begin{array}{l}0 \\
2\end{array}$ & 0 & 2 & 2 & 2 & $\mathrm{O}^{8} \mathrm{O}^{7}-\mathrm{C}^{6} \mathrm{O}=\mathrm{O}^{12} \mathrm{O}^{10} \mathrm{O}^{11}$ \\
\hline$E_{8}\left(a_{2}\right)$ & 2 & 2 & $\begin{array}{l}2 \\
0\end{array}$ & 2 & 0 & 2 & 2 & $100-0^{2}$ \\
\hline$E_{8}\left(a_{1}\right)$ & & 2 & $\begin{array}{l}2 \\
0\end{array}$ & 2 & 2 & 2 & 2 & \\
\hline$E_{8}$ & 2 & 2 & $\stackrel{2}{2}$ & 2 & 2 & 2 & 2 & 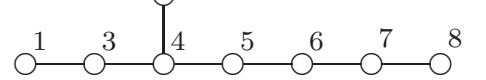 \\
\hline
\end{tabular}

Appendix B. The exceptional root systems in GAP

In this appendix we list the positive roots of the exceptional root systems, in the order in which they appear in GAP4. The tables have to be read from left to right, and from top to bottom. So the first root is the one top left, the second root is the second one on the first line, and the last root is the one bottom right. For each root its coefficients with respect to a basis of simple roots are given.

The roots for $F_{4}$ in Table 8 may seem slightly strange. This is due to the fact that in GAP4 the positive roots are ordered differently than usual. In this table the coefficients of each root with respect to the "usual" ordering of a basis of simple roots is given (i.e., as in [1] ). However, the roots are listed in the same order as they are in GAP4.

Table 7: Positive roots in the root system of type $G_{2}$.

\begin{tabular}{|l|l|l|l|l|l|}
\hline 10 & 01 & 11 & 21 & 31 & 32 \\
\hline
\end{tabular}

Table 8: Positive roots in the root system of type $F_{4}$.

\begin{tabular}{|l|l|l|l|l|l|l|l|}
\hline 0001 & 1000 & 0010 & 0100 & 0011 & 1100 & 0110 & 0111 \\
1110 & 0120 & 1111 & 0121 & 1120 & 1121 & 0122 & 1220 \\
1221 & 1122 & 1231 & 1222 & 1232 & 1242 & 1342 & 2342 \\
\hline
\end{tabular}


Table 9: Positive roots in the root system of type $E_{6}$.

\begin{tabular}{|c|c|c|c|c|c|c|c|c|}
\hline 0 & 1 & 0 & 0 & 0 & 0 & 0 & 1 & 0 \\
10000 & 00000 & 01000 & 00100 & 00010 & 00001 & 11000 & 00100 & 01100 \\
0 & 0 & 0 & 1 & 1 & 0 & 0 & 1 & 0 \\
00110 & 00011 & 11100 & 01100 & 00110 & 01110 & 00111 & 11100 & 11110 \\
1 & 1 & 0 & 1 & 0 & 1 & 1 & 1 & 1 \\
01110 & 00111 & 01111 & 11110 & 11111 & 01210 & 01111 & 11210 & 11111 \\
1 & 1 & 1 & 1 & 1 & 1 & 1 & 1 & 2 \\
01211 & 12210 & 11211 & 01221 & 12211 & 11221 & 12221 & 12321 & 12321 \\
\hline
\end{tabular}

Table 10: Positive roots in the root system of type $E_{7}$.

\begin{tabular}{|c|c|c|c|c|c|c|}
\hline 0 & 1 & 0 & 0 & 0 & 0 & 0 \\
100000 & 000000 & 010000 & 001000 & 000100 & 000010 & 000001 \\
0 & 1 & 0 & 0 & 0 & 0 & 0 \\
110000 & 001000 & 011000 & 001100 & 000110 & 000011 & 111000 \\
1 & 1 & 0 & 0 & 0 & 1 & 0 \\
011000 & 001100 & 011100 & 001110 & 000111 & 111000 & 111100 \\
1 & 1 & 0 & 0 & 1 & 0 & 1 \\
011100 & 001110 & 011110 & 001111 & 111100 & 111110 & 012100 \\
1 & 1 & 0 & 1 & 1 & 0 & 1 \\
011110 & 001111 & 011111 & 112100 & 111110 & 111111 & 012110 \\
1 & 1 & 1 & 1 & 1 & 1 & 1 \\
011111 & 122100 & 112110 & 111111 & 012210 & 012111 & 122110 \\
1 & 1 & 1 & 1 & 1 & 1 & 1 \\
112210 & 112111 & 012211 & 122210 & 122111 & 112211 & 012221 \\
1 & 1 & 1 & 2 & 1 & 1 & 2 \\
123210 & 122211 & 112221 & 123210 & 123211 & 122221 & 123211 \\
1 & 2 & 1 & 2 & 2 & 2 & 2 \\
123221 & 123221 & 123321 & 123321 & 124321 & 134321 & 234321 \\
\hline
\end{tabular}

Table 11: Positive roots in the root system of type $E_{8}$.

\begin{tabular}{|c|c|c|c|c|c|}
\hline 0 & 1 & 0 & 0 & 0 & 0 \\
1000000 & 0000000 & 0100000 & 0010000 & 0001000 & 0000100 \\
0 & 0 & 0 & 1 & 0 & 0 \\
0000010 & 0000001 & 1100000 & 0010000 & 0110000 & 0011000 \\
0 & 0 & 0 & 0 & 1 & 1 \\
0001100 & 0000110 & 0000011 & 1110000 & 0110000 & 0011000 \\
0 & 0 & 0 & 0 & 1 & 0 \\
0111000 & 0011100 & 0001110 & 0000111 & 1110000 & 1111000 \\
1 & 1 & 0 & 0 & 0 & 1 \\
0111000 & 0011100 & 0111100 & 0011110 & 0001111 & 1111000 \\
0 & 1 & 1 & 1 & 0 & 0 \\
1111100 & 0121000 & 0111100 & 0011110 & 0111110 & 0011111 \\
1 & 1 & 0 & 1 & 1 & 1 \\
1121000 & 1111100 & 1111110 & 0121100 & 0111110 & 0011111 \\
0 & 1 & 1 & 1 & 0 & 1 \\
0111111 & 1221000 & 1121100 & 1111110 & 1111111 & 0122100 \\
1 & 1 & 1 & 1 & 1 & 1 \\
0121110 & 0111111 & 1221100 & 1122100 & 1121110 & 1111111 \\
1 & 1 & 1 & 1 & 1 & 1 \\
0122110 & 0121111 & 1222100 & 1221110 & 1122110 & 1121111 \\
\hline
\end{tabular}


Computing with nilpotent orbits in simple Lie algebras of exceptional type

\begin{tabular}{|c|c|c|c|c|c|}
\hline \multicolumn{6}{|c|}{ Table 11 (continued). Positive roots in $E_{8}$} \\
\hline 1 & 1 & 1 & 1 & 1 & 1 \\
0122210 & 0122111 & 1232100 & 1222110 & 1221111 & 1122210 \\
1 & 1 & 2 & 1 & 1 & 1 \\
1122111 & 0122211 & 1232100 & 1232110 & 1222210 & 1222111 \\
1 & 1 & 2 & 1 & 1 & 1 \\
1122211 & 0122221 & 1232110 & 1232210 & 1232111 & 1222211 \\
1 & 2 & 2 & 1 & 1 & 1 \\
1122221 & 1232210 & 1232111 & 1233210 & 1232211 & 1222221 \\
2 & 2 & 1 & 1 & 2 & 2 \\
1233210 & 1232211 & 1233211 & 1232221 & 1243210 & 1233211 \\
2 & 1 & 2 & 2 & 2 & 1 \\
1232221 & 1233221 & 1343210 & 1243211 & 1233221 & 1233321 \\
2 & 2 & 2 & 2 & 2 & 2 \\
2343210 & 1343211 & 1243221 & 1233321 & 2343211 & 1343221 \\
2 & 2 & 2 & 2 & 2 & 2 \\
1243321 & 2343221 & 1343321 & 1244321 & 2343321 & 1344321 \\
2 & 2 & 2 & 3 & 3 & 2 \\
2344321 & 1354321 & 2354321 & 1354321 & 2354321 & 2454321 \\
3 & 3 & 3 & 3 & 3 & 3 \\
2454321 & 2464321 & 2465321 & 2465421 & 2465431 & 2465432 \\
\hline
\end{tabular}

\section{References}

1. N. Bourbaki, Groupes et Algèbres de Lie (Hermann, Paris, 1968) chapters 4, 5 and 6. 294

2. R. W. CARTer, Finite Groups of Lie Type. Conjugacy classes and complex characters (Wiley \& Sons, Chichester, 1985). 281, 283

3. David H. Collingwood and William M. MCGovern, Nilpotent orbits in semisimple Lie algebras, Van Nostrand Reinhold Mathematics Series (Van Nostrand Reinhold, New York, 1993). 281, 282

4. J. Dixmier, Algèbres Enveloppantes (Gauthier-Villars, Paris, Bruxelles, Montréal, 1974). 281

5. D. Panyushev, A. Premet and O. Yakimova, 'On symmetric invariants of centralisers in reductive Lie algebras', J. Algebra 313 (2007) 343-391. 281

6. Dmitri I. Panyushev, 'The index of a Lie algebra, the centralizer of a nilpotent element, and the normalizer of the centralizer', Math. Proc. Cambridge Philos. Soc. 134 (2003) 41-59. 281

7. V. L. Popov, 'The cone of Hilbert null forms', Tr. Mat. Inst. Steklova 241 (2003) 192-209 (Russian); English transl. Proc. Steklov Inst. Math. 241 (2003) no. 2, 177-194. 284

8. R. W. Richardson, 'Commuting varieties of semisimple Lie algebras and algebraic groups', Compositio Math. 38 (1979) 311-327. 285

9. Jirō SEkiguchi, 'A counterexample to a problem on commuting matrices', Proc. Japan Acad. Ser. A Math. Sci. 59 (1983) 425-426. 281, 285

10. E. B. Vinberg and O. S. Yakimova, 'Complete families of commuting functions for coisotropic Hamiltonian actions', Preprint, 2005, arXiv:math/ 0511498 v2 [math.SG]. 281 
Computing with nilpotent orbits in simple Lie algebras of exceptional type

11. O. S. Yakimova, 'The index of centralizers of elements in classical Lie algebras', Funktsional. Anal. i Prilozhen. 40 (2006) 52-64, 96. 281

Willem A. de Graaf degraaf@science.unitn.it

Dipartimento di Matematica

Università di Trento

via Sommarive 14

I-38100 Povo (Trento)

Italy 